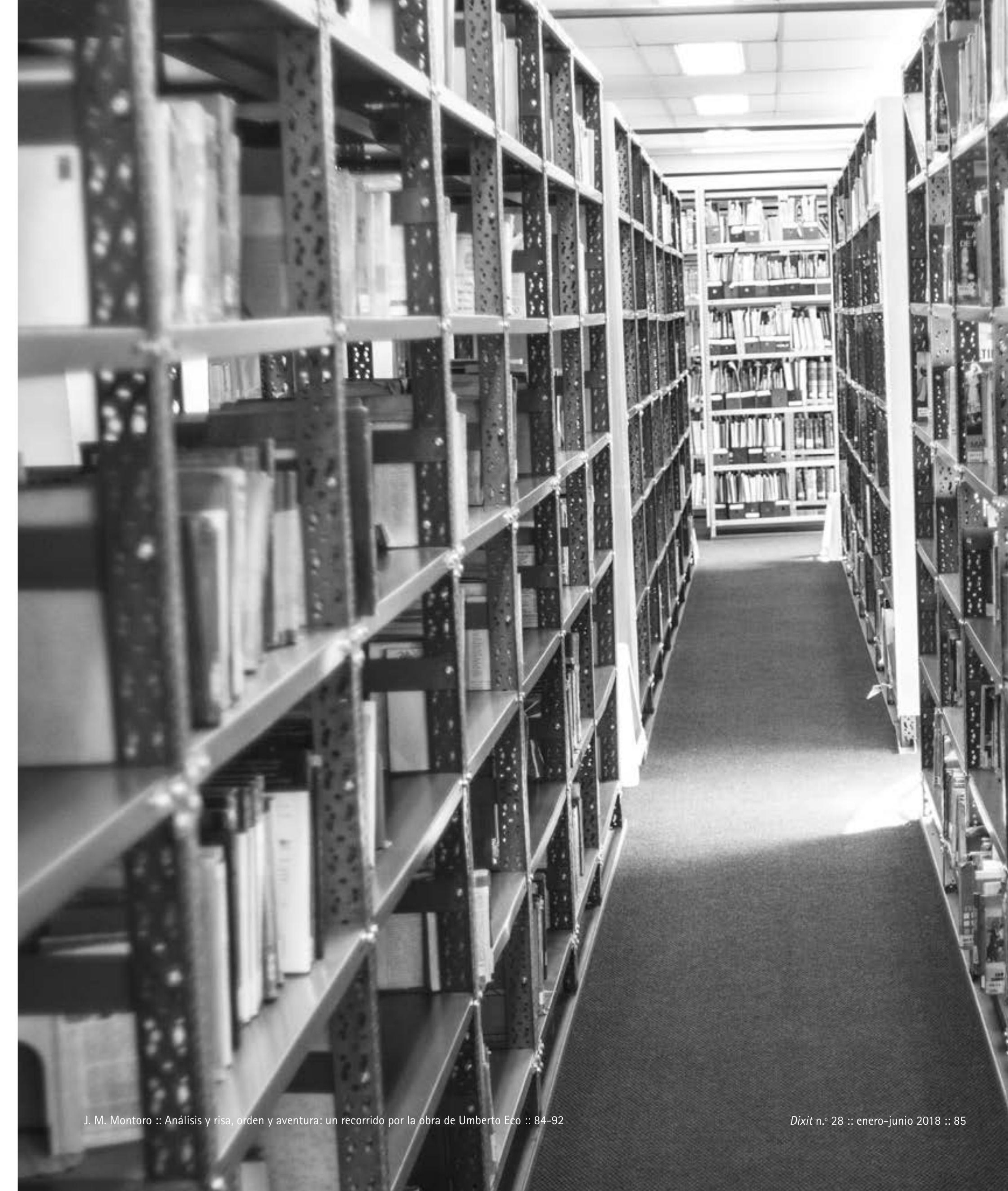


Libro reseñado ¿Qué es la semiótica? Existe una definición profunda, sintética, fácil de entender, fiel a las complejidades que retóricas del campo: una teoría de la mentira. (1932-2016), quien la desarrolla apelando a las bases de la lógica: algo que no sirve para mentir, dice, tampoco sirve para decir una verdad y de hecho no sirve para decir nada (Eco, 1988, p. 28). Una mentira, entonces, crea un mundo paralelo y heterogeneo que muestra un objeto de manera distinta a como el mundo nuestro, el de la verdad, lo interpreta. Así también funciona los signos: un signo es algo que está en lugar de un objeto y dice algo acerca de él.

Claudio Paolucci (2017) elige introducir su libro-homenaje a su gran mentor desglosando esta famosa definición y problematizando cómo ese universo de mentira se relaciona con el de la ficción. Fste libro da nicio a una serie de la casa editorial Feltrinelli, denoinada Eredi porque busca captar la obra de grandes telectuales a partir de las palabras de sus herederos cos che sais, de hecho, ha trabajado en conjunto con muchos e importantes pensadores, pero probablemente Paolucci sea el semiògo los últis ses vi procupado por extender su legado subtitulo, el libro tiene la doble virtud de ordenar la inmensa obra de Eco mediante ejes claros, articulados coherentes y a la vez presentar las aventuras y detalles críticos para entender su pensamiento.

Dentro de este esfuerzo por ordenar, otra virtud de pro es desmitificar la figura de Eco como un erudito, polimata o, simplemente, el hombre que sabia todo com mucho se lo recordó desde su muerte. Paolucci afirm para mostrar una figura de sí mismo muy distinta de que la opinión pública y los medios reflejaban. Lejos d intelectual deseoso de saber por saber, Eco entendió . corrió por carriles independientes: Paolucci entiente que no hubo un Eco medievalista, un Eco semiólogo, un Eco escritor y un Eco ensayista, desconectados el uno del otro, sino un único filósofo que anidó y distribuyó su trabajo en función de sus objetivos y las características del objeto a abordar.

Esto queda de manifiesto al repasar la estructura de libro, que cuenta con diez capitulos además de un prólogo, una introducción y una emotiva despedida. La sucesion busca trazar una biografía intelectual que parte su etapa de estudiante (“La formazione") y, a partir de algunas publicaciones clave, se adentra en su primera etapa académica: la pre-semiótica ("Opera aperta", "Inermezzo" “Apocalittici e integrati”). Después, Paoluc acentúa la etapa semiótica y narrativa ("La guerriglia semiológica", "L'Enciclopedia e la svolta semiotica", "I iso e la rosa"). Por ultimo, plantea similitudes entre su obra narrativa y su obra semiótico-filosófica (" $\mathrm{Pa}$ rallelismi , para cerrar con un resumen integral de su pensamiento, a la luz del recorrido presentado, que Di cio di cri nou si pus de su obra ("Quid sit veritas.

Una de las constantes a lo largo del libro es la tripartCoin de actividades intelectuales a partir de la cual $\mathrm{E}$. habría organizado sus ejes de investigación y todal Eco producción: la historia, la teoría y la narración. Desde sus años de formación, Eco se autodefinía como "u historiador de la cultura", en tanto la historia es aque dominio que nos permite identificar los problemas y hace las preguntas adecuadas. La teoría, por su parte, viene dar las respuestas de manera metódica, pero con algunos limites intinsecos. La narración, por ultimo, permite a los problemas planteados. Para Paolucci, la relación entre teoría y narración es similar a la que mantienen el

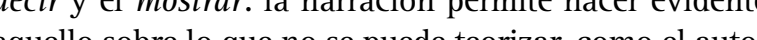
entende que ocurre con El Nombre de la Rosa (1980).

Los dominios del conocimiento, subraya Paolucci, funcionan en Eco como grandes campos de reglas y códigos que dan forma a la materia amorfa, un aspecto que aparece ya presente en Obra Abierta (1962). No obsante, es necesario salir de ellos y entender aquello que los rodea para resolver problemas intelectuales. De la misma manera que hace falta echar mano a la narración para resolver problemas de la teoría, y que, siguiendo e ifulo de su ultima gran obra (Eco, 1997), solo a través "no es posible entender Mike Bong on on an entender "Husserl y la fenomenologí. No es posible entender Superman y la literatura de masas sin entender Marx y Gramsci" (Paolucci, 2017, p. 56) 2. Lo novedoso del planteo de Eco, según Paolucci, es que estas causalidades son más que un postureo intelectual de explicar lo bana on lo teorico, y funcionan como relaciones reciprocas que corren en doble sentido: el ornitorrinco ecesario para entender la filosofia de Kant.

Entender la cultura como una enciclopedia es quizás la principal huella del pensamiento del Eco maduro, y su ni Wikipedia. Según el autor. Eco cré una nueva cartografía del saber donde todos los dominios del conocimiento se encuentran sin jerarquía ni un centro que los organice. Esa enciclopedia es contradictoria y laberíntica, y no hay quien pueda apropiársela en su totalidad. Más bien nuestra competencia como usuarios consiste en detentar secciones parciales e interconectadas. Eco atribuye una "tercera dimensión" a esa enciclopedia, reconocer las relaciones entre cultura alta y cultura esos saberes a la luz de los usos sociales.
Aquí Paolucci muestra una toma de partido muy clara por parte de Eco. Para este último, la cultura para garantizar su hegemona en le producion de conocimiento. El intelectual debe asumir entonces una actitud de militancia y operar como un educador que oriente la lectura crítica y acompañe las audiencias a reconocer las contradicciones y a desnaturalizar esta construcción desde el poder.

Según Paolucci, la militancia en Eco es clave para enter su rol activo en los medios de comunicación sus columnas en prensa y hasta su propia elevación como refrente en la sociedad italiana y la cultura (desgloses críticos de la cultura popular en ougica" a teórico que asigna a la interpretación un poder mayor que la mera recepción y depósito de información, rol que le había reservado la Teoría Matemática de la Información. Como puede verse a partir de Lector in fabula (1981), en el Eco maduro la interpretación es un proceso que necesita la cooperación del lector para activar una serie de significados que pueden no ser los deseados por el productor.

Sería interesante, en ese sentido, oponer la militanci de Eco -hija de su propia reflexión teórica- a otros Chomsky. Una de las primeras cosas que se le dice a un estudiante de Lingüistica es que el Chomsky científico y lingüista tiene poco que ver con el militante politico y viceversa. No es posible reconstruir una teoría desde la otra, porque las motivaciones y preocupaciones del intelectual difieren cuando encara su labor cientifica y cuando asume un rol de critica politica. En el caso de Eco, la interconexión entre producción académica y militancia ensayistica siguen un mismo patrón de coherencia como la teor
2:: Las citas textuales
que aparecen en esta. reseña fueron traducidas 
Naturalmente, el movimiento opuesto a la elevazione es la caduta (caída) de la cultura alta y, de igual modo que en

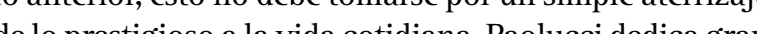
parte del libro a explicar y desarrollar de qué manera se da este movimiento. Para Eco, la risa sería el meconismo que permite desacralizar el orden dado.

Paolucci es bastante contundente al respecto y en distintos pasajes de su libro demuestra cómo Eco considera a la risa como "el arma de la filosofia" (2017, p. 153) que permite "superar la duda cartesiana" (p. 162) porque muestra el carácter provisorio de lo que en un momento y en un lugar se cree verdadero. El final de El Nombre de la Rosa es contunde veristencia de un comedia habitán a sus lectores a rír de cosas seras como las sagradas escrituras, por lo que es considerado perverso para la cultura alta y por lo tanto merece ser destruido a los ojos de sus instituciones.

No es menor el hecho de que, para Eco, uno solo pueda reirse de algo cuando pertenece a aquello de lo se rie. Reirse es salirse de ese sistema, pero para poder sali primero hace falta conocerlo y formar parte. A partir de esta idea es que, en lo personal, no me satisface la solución de la risa como dispositivo de caida de la cultura ca. Quiero ser nuy criśsoso en esto, porque Paoluce lectual de Eco y, por ende, es muy del ada la line entre discutir con su interpretación y discutir con la filosofí misma del maestro. En cualquier caso, tampoco ese es el propósito de estas líneas. Pero, en definitiva, si para desacralizar un elemento hace falta reírse de él, y si para reirse de algo hace falta pertenecer a ello, la caduta es un movimiento reservado solo para quienes ya integra una élite intelectual.

Paolucci comenta el gusto que Eco sentía por Rabelais, en tanto su Gargantúa y Pantagruel encarnaba esta ac- titud de risa frente a la naturaleza humana, considerad desde siempre un valor de la cultura alta. ¿Pero no es un producto de la cultura alto? ¿Por qué un humanista francés del siglo XVI y no las Cophas del Prouincil que se burlaban de las autoridades castellanas del siglo XV? El orden dado no es estático, y si la risa y la caída funcionan desde el interior de un sistema, jpodemos reirno de un tiempo, un lugar, una clase social o una situación a la que no pertenecemos?

Paolucci (o Eco mismo) quizás respondería que todo depende de las porciones más densas que cada uno encuentre en sus comptencias enciclopédicas. Y, asi, mi to mestituiria, Francisco Acuña de Figueroa letrista ya no de ujo de de dos himnos nacionales

Pero, si lo comparamos con el movimiento contrario, la elevación permite echar una mirada crítica sobre objeos mundanos independientemente de la porción de la enciclopedia a la que pertenecen. Puedo no conocer $\mathrm{Su}$ perman, pero el analisis me muestra como se construye cormo se ongina su naturalización en la cultura populary, seducción La concér sus procesos de manipulacióny intelectual de Eco: se hase con el texto pe la mulitine la masa, enseñarle los mecanismos que operan detrís a la fachada de lo simple y aumentar la capacidad de análisis de los individuos.

La caída entendida como risa no tendría un componente análogo, porque solo me puedo reír con quienes comparten, siguiendo a Sperber y Wilson (1995), las misma implicaciones que marcan la relevancia pragmática de 1 a situación cómica. Quizás la risa como actitud filosófica supera a la duda cartesiana porque ataca a la verdad (en lugar de solamente no-defenderla) y muestra su valor
3:: Acuña de figureaa
(1791-1862) les conocido por ser el autor de la de Uruguay, aunque
también escribio el temática evocada y sus contextos de uso,
los himnos nacionales pertenecen a porcion
del la cultura alta. Sin embargo, es de apreciar que Acuña de una muy amplia serie
de contextos lenguies propósitios y temas (ver 
parece un mecanismo limitado para derribar la autoridad simbólica de la cultura alta y acortar la brecha de poder

Con todo, ¿existe algún dispositivo que pueda ocupa ese rol de la caída? Elaborar tal respuesta requeriría de un ensayo que la justifique, y eso trasciende los objetivos de esta reseña crítica. Solamente me detengo en este tema porque es un punto que Paolucci trabaja con
profundidad, y es una de las claves de lectura con la que este autor propone dar sentido a la prolíica trayectoria de Eco. Y yo, que fui alumno de Paolucci, busco hacer mismo que él aprendio -y me enseño- de su maestro criticar las ideas del professore mediaciones posibles (p. 36).

Si, como dice Paolucci, justamente por las limitaciones del nivel de la teoría no es posible escribir un "tratado
general de la risa", las dos obras más importantes de Eco que no dicen pero muestran su funcionamiento como mecanismo de caída de la cultura alta son Diario Minimo (1963), en el ámbito del ensayo y la columna satírica, El nombre de la rosa como novela. En ambos casos humor ocupa un lugar central, ya sea por lo enunciad como foco de la problematica o por la enunciación de la voz narrativa que busca activar ese efecto (Colón descubriendo America en transmision televisada en vivo, un informe antropologico que relata con estupor las formas imaginar a Diderot vendiendo enciclopedias puerta puerta, etcétera).

Es humorístico, es cierto, pero por sobre todas las cosas plantea una inversión del punto de vista. En Cuore, de Edmondo De Amicis, debemos valorar a Franti, el antagonista que rie en lugar de aceptar una disciplina protofascista (Eco, 1975/2016, pp. 81-92). La sátira de Lolita de Nabokov, es Nonita porque la obsesión no es por las jovenes, sino por las ancianas (pp. 11-17). En otro relato, exotismos de la cultura "civilizada" de Milán (pp. 63-80). en El nombre de la rosa, Eco mismo no se autorretrata serí congrugon a terí como buscaba-, sino en el aprendiz Adso de Melk. De la misma manera que Borges, una figura admirada y modelizada por Eco, inspira al antagonista Jorge de Burgos, un monje maligno incapaz de tolerar la risa. Sí, Borges: el mismo que se réa de Cervantes, Judas y Lucrecio.

La pregunta queda planteada: ¿será el cambio de punto de vista $-\mathrm{y}$, con él, la capacidad de revertir la cultura alta- otra forma de instrumentar el movimiento de la caida en la enciclopedia del saber compartido? Si asi fuera, tal vez deberiamos buscar en la enunciación y no en la risa estrictamente la clave del enroque. En otras idades, sería aquello que habilita el cambio lo que genera la caída y no el efecto de reconocer al principe vestido en harapos. En eso creo que la teoría de la cultura de Eco recuperaría parte de su caracterización semiótica: es la función signica y su intercambiabilidad en el sistema la que habilita la caida, más que su relación inferencial con la cultura alta de la enciclopedia.

En la última parte del libro, Paolucci trabaja con detalle la relación entre signo y verdad, y muestra una dimensión poco explorada en el pensamiento de Eco, particularmente

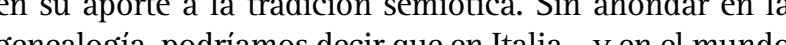
latino en general- se atribuye a Eco la llegada de Peirce a la bibliografia semiótica. Si, como se afirma comúnmente, la semiótica estudia los sistemas y procesos de significación, el estructuralismo francófono y sus extensiones en el resto de Europa continental y America Latina se preocuparon por trabajar con los sistemas. El posestructuralismo, desde luego, discutió esa idea, pero atacando la solidez y omnipotencia que gozaban esas estructuras. Eco se introdujo en la semiotica desde el estructuralismo, pero con los

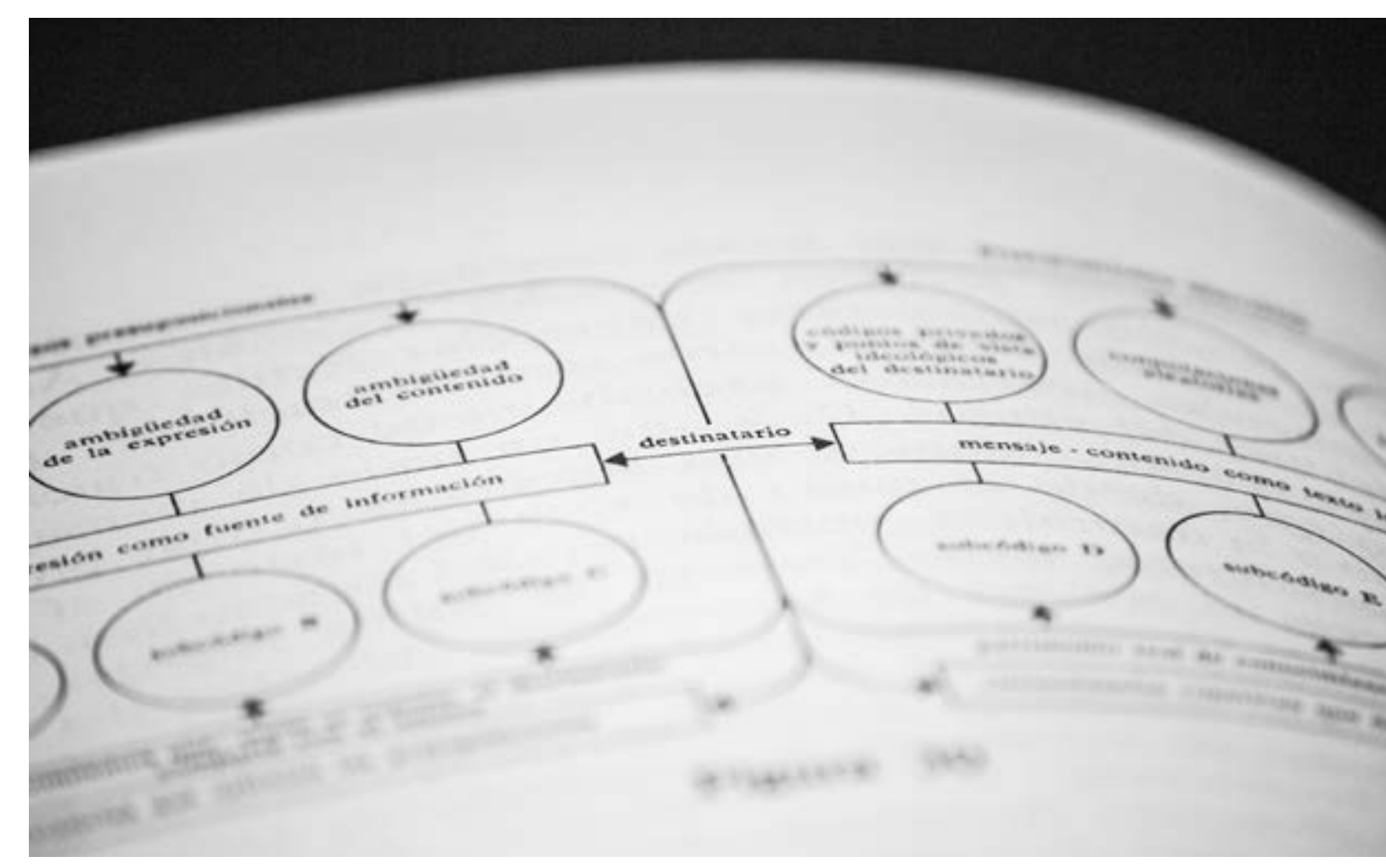

proceso productivo donde las inferencias a partir de los "la división maniquea entre verdad y falsedad" (2017, p. signos observables son fundamentales para la creación de 152). Paolucci llama a esto la "pericia semiótica" porque sentido. Uno conoce porque el signo, además de asociar no hay una verdad que suplante a otra, sino distintas sonido), proyecta una tercera vía que Peirce denominó puto incial: pra Ecol conocimiento sería un instrinterpretante. Y ese interpretante actúa iniciando un nuevo mento práctico más que un conjunto de conocimientos proceso, análogo al anterior, y construye así una cadena Saber no es saber cosas; es saber cómo encontrarlas en de signos hacia el infinito.

Saber no es saber
medio minuto.

Peirce, como buen logico, considera que esa cadena de sen- El libro trabaja la trayectoria de Eco de manera extido se da en forma lineal. El interés de Eco, afirma Paolucci, haustiva, a mi entender, cubre y sintetiza muy bien es demostrar por el contrario que el conocimiento y, sobre las principales problemáticas. Trabaja su perspectiv todo, el acceso a la verdad se dan a través de la fuerza del de semiölogo y la encuadra dentro del marco genera falso": al igual que ocurre con las ciencias, no conocemos de su pensamiento, de modo que se puede observar acumulando información en progresión continua, sino como los intereses confluyen y como se instrumen-

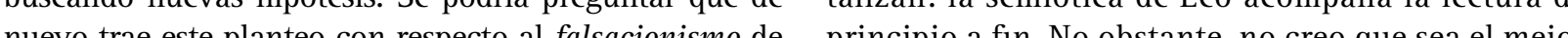
Popper. El autor no plantea esa pregunta, pero defiende libro para alguien a quien le interese erclusivamente y categóricamente la idea de que para Eco lo interesante no en detalle la obra semiótica de Eco. Dado el enfogue, está en el resultado sino en el proceso, como la escalera temas como las condiciones de la traducción, los límites que descartamos cuando subimos a la cima. de la interpretación, la relación entre signo, experiencia y memoria, y su antropologia semiotica no aparecen Para muestra, Paolucci afirma que los libros favoritos de particularmente desarrollados. El propio libro sugier Eco en su biblioteca personal eran todos sobre visiones las lecturas de Lorusso (2008) si uno busca un enfoque del mundo que se creyeron validas en algún momento y basado en la teoría de Eco y de Trainito (2011) si se quiere hoy nos parecerian ridiculas. Ese camino es el que permite especializar en la narrativa equiana, ambos polos de los el estado actual de la cultura y, por eso, más de allá de la que Paolucci se ubica en un punto intermedio. En 


\section{Los pequeños}

obra colectiva Umberto Eco In His Own Words (2017) o Referencias

la lectura de Cogo (2010) para evaluar el rol de Umberto Armand Ugón, P. (2015). Versificar para el común en el siglo XIX: Los epigramas de Francisco Acuña de Figueroa. Cuadernos encontrado bibliografía especializada en español.

Se aprecia que el lector modelo del libro de Paolucci son aquellos que han oido escuchar de Eco yla semiótica, pero no han profundizado, ya que la terminologia técnica es casi nula. De todos modos, el libro está a la altura de un curso universitario de semiótica interpretativa, lo cual es mérito del autor. El estilo es fluido, coloquial y por mo-

Al tratarse de un libro homenaje, Paolucci muestra en todo momento un gran cariño y una cercanía especia con Eco a través de anécdotas de sus años de estudiansemiólogo. En el plano estilistico, esto le da humanidad y frescura al libro, que aplica en el campo una de las enseñanzas: Paolucci alli muestra aspectos de Eco como mentor y amigo que claramente no se podrian decir. Por contrapartida, la despedida ( Congedo" que cierra el libro se explicita como una instancia intima con el maestro y presenta un dramatismo que, a mi gusto, es excesivo. Quizá falta una perspectiva más crítica a la propia ferencia a qué. elementos de su temprana producció académica discutieron con la obra contemporánea de su profesor, en particular en torno a los problemas que Eco planteó en Kant y el ornitorrinco (1997) y que Paolucci profundizó en su primer libro, Strutturalismo e interpretazione (2010). Este pasaje está presentado más bien a modo de anécdota para ilustrar la generosidad y apertura de Eco a recibir insumos valiosos de sus estudiantes, pero quizás seria interesante evalua que desalios dejó plantudos, cómo se pueden segu explorando y qué caminos se pueden recorrer.
C. (2010). Fenomenologia di Umberto Eco. Bolonia, Italia Baskerville.

Eco, U. (1988). Tratado de Semiótica General. Barcelona, España: Lumen.

Eco, U. (1997).Kantyelomitorrinco. Barcelona, España: Lumen. . (2016), Diario Minimo. Milán, Italia: Bompiani (Trabajo original publicado en 1975)

ser. A. M. (2008). Umberto Eco: tert Italia: Bompiani. Italia: Feltrinell.

Solari, C (20 de febrero de 2016). Umberto Eco, el hombre que sabia todo. Una lista [Entrada en un blog]. Recuperado de https://hipermediaciones.com/2016/02/20/umbertoeco-el-hombre-que-sabia-todo-una-lista/

Sedda, F. (7 de abril de 2016). L'essere e l'enciclopedia. Forme del realism e della cultura nell'opera semiotica di Umdi Studi Semiotici. Recuperado de http:I/www.academia edu/24161429/Lessere_e_lenciclopedia._Forme_del_reato_Eco

rber, D., y Wilson, D. (1995). Relevance: Communication and Cognition. Oxford, Reino Unido: Blackwell.

Works of Umberto Eco. E/C Rivista digitale dell'Associt cordlD=687\&kT_download 1 =e649dbeobb8d2337d270ab929585533

Thellefsen, T., y Sørensen, B. (Eds.). (2017). Umberto Eco In His Own Words. Boston, MA: De Gruyer Mouton.

ssea nella biblioteca d Babele. Saonara, Italia: II Prato.
(2010). Strutturalismo e interpretazione. Milán, berto Eco. Elc. Rivista digitale dell'Associazione Italiana zione Italiana di Studi Semiotici. Recuperado de http. www.ec-aiss.t//includes/tng/pub/tNG_download4.php?re-
Ilustración de Virginia Daglio Ksiazenicki

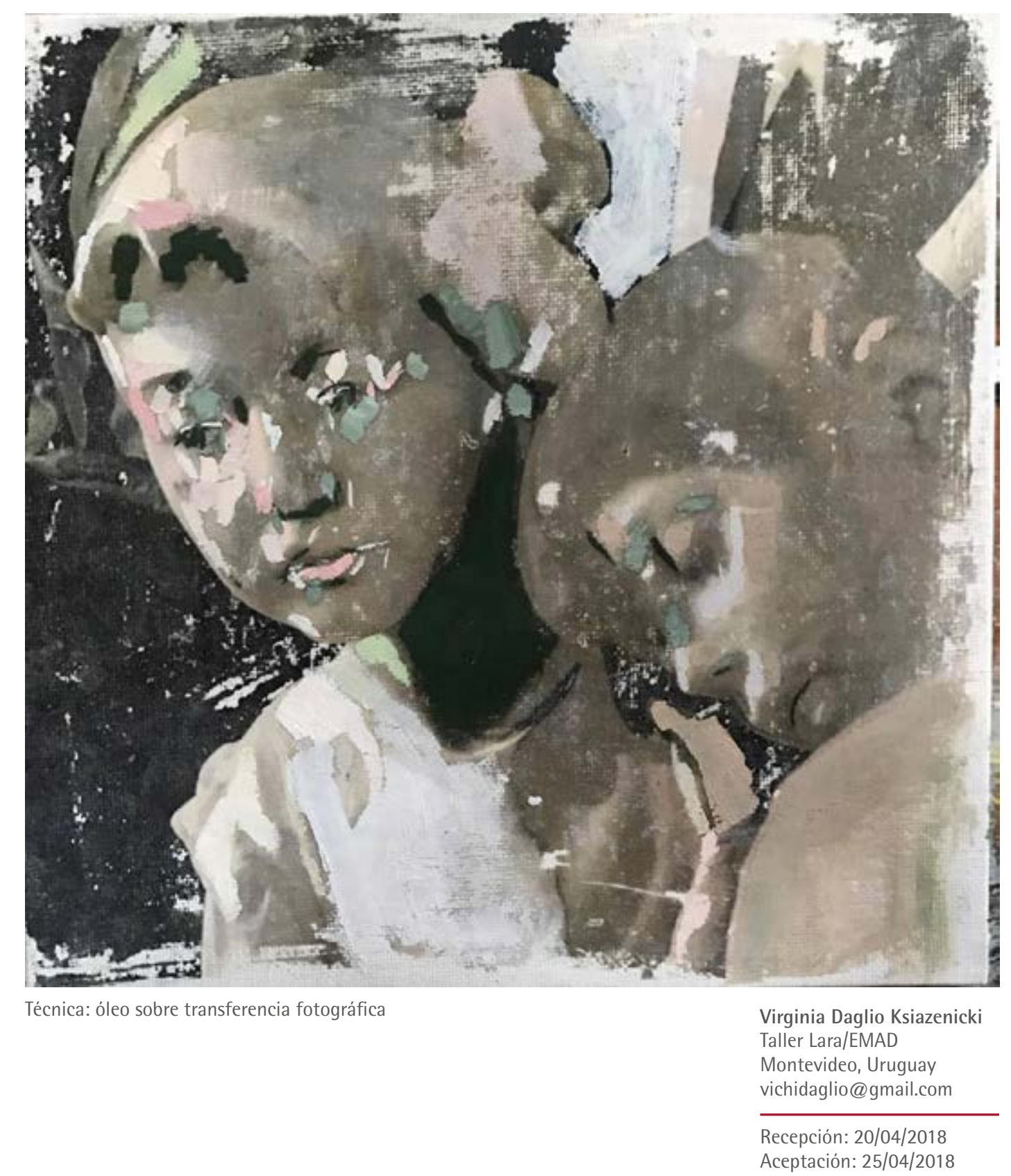

